\title{
MEEMD Decomposition-Prediction-Reconstruction Model of Precipitation
}

\author{
Jian Liu \\ Hunan University \\ Rong Li \\ Hunan University \\ Xinyu Suo \\ Hunan University \\ EnHui Lu \\ Hunan University
}

Yongtao Wang ( $\nabla 409011805 @ q q . c o m$ )

Hunan University https://orcid.org/0000-0001-6281-5377

\section{Research Article}

Keywords: MEEMD, PSO-SVM, CNN, RNN, Forecast

Posted Date: February 23rd, 2022

DOI: https://doi.org/10.21203/rs.3.rs-1383374/v1

License: (c) (1) This work is licensed under a Creative Commons Attribution 4.0 International License. Read Full License 
3 Abstract: Aiming at the low prediction accuracy of precipitation time series data,

4 proposes an Modified Ensemble Empirical Mode Decomposition-prediction

5 -reconstruction model(MDPRM). First, The non-stationary precipitation time series is

6 decomposed into multiple decomposition terms by MEEMD. Then, according to the

7 characteristics of different decomposition items, the particle swarm optimization

8 support vector machine (PSO-SVM), convolutional neural network (CNN) and

9 recurrent neural network (RNN) models are used for prediction respectively. Finally,

10 the prediction results of each decomposition item are superimposed and reconstructed

11 to form the final prediction results. The summer precipitation in the Wujiang River

12 Basin in Guizhou Province from 1961 to 2018 was used as an example to verify the

13 results. The results show that:compared with BP, SVM, RNN and other prediction

14 methods, the MDPRM proposed in this paper has higher prediction accuracy, and can

Yongtao Wang

409011805@qq.com

Jian Liu

liujian@hnu.edu.cn

Rong $\mathrm{Li}$

jyylirong@hnu.edu.cn

Xinyu Suo

347359354@qq.com

EnHui Lu

1181995024@qq.com

${ }^{1}$ State Key Laboratory of Advanced Design and Manufacture for Vehicle Body, Hunan University, Lushan South Road, Yuelu District, Changsha, Hunan Province, 410082, China

${ }^{2}$ Guizhou Institute of Water Resources Science, Guizhou Guiyang 550002, China 
effectively guide the regional water resources allocation and scheduling, drought relief and disaster reduction.

Keywords: MEEMD·PSO-SVM.CNN·RNN·Forecast

\section{Introduction}

Precipitation prediction can scientifically and rationally guide the planning, development and distribution of regional water resources, reduce floods and drought disaster losses, alleviate water shortages and other issues ; The prediction results can provide reference for water supply and demand analysis, planning and resources management.The research results of Chen Jing et al. show that: (1) May to September are the months when extreme precipitation events occur in Guizhou, especially in June and July. (2) The extreme precipitation events in Guizhou tend to be more and stronger, and the proportion of extreme precipitation to total precipitation tends to increase. (3) The overall change trend of the annual number of rainstorm days is not obvious and shows a relatively obvious downward trend since the 21 st century. (4) The amount of heavy rainfall is on the rise as a whole, and it has shown a relatively obvious decreasing trend since the beginning of the 21st century, but the proportion of heavy rainfall is on the rise (Chen et al. 2015). By analyzing the precipitation data in the Wujiang River Basin, it is found that the average precipitation in the summer of 1961-2018 (June-August) accounted for $45.16 \%$ of the annual average precipitation, which was the main part of the annual precipitation.Meanwhile,from June to August was the months when extreme precipitation is concentrated.It is of great significance to effectively guide the water resources dispatching management and safe flood control work during the summer flood period (Li and Xue 2018).

\section{MEEMD Principle}

\subsection{EMD Method}


The EMD Method decomposes the signal according to the time scale characteristics of the data itself, it does not need to set any basis function in advance. This characteristic makes the EMD method theoretically applicable to the decomposition of any type of signal,it suitable for analyzing nonlinear and non-stationary signal sequences, and has a high signal-to-noise ratio. Its steps are as follows (Cheng et al. 2018; Jiang et al. 2016; Qi et al. 2016):

(1) For any signal $s(t)$, determine the extreme points $s(t)$ firstly, and then connect all the maximum and minimum points with a curve respectively.

(2) Take these two curves as the upper and smaller envelopes of $s(t)$ respectively. The mean value of each point on the upper and smaller envelopes is denoted as $m$, and the difference between $s(t)$ and $m$ is denoted as $h$, then $h=s(t)-m$.

(3) Consider $h$ as a new $s(t)$, and repeat the above operations. When $m$ is zero or close to zero, $h$ is small enough, or $h$ is one of the monotonic functions, denote $c 1=h$. Treat $c 1$ as $I M F_{1}(t)$ and write $s(t)-c 1=r_{1}(t)$

(4) Treat $r_{1}(t)$ as the new $s(t)$ and repeat the above process. Get $I M F 2(t), \quad c 2, \quad r 2(t), \quad I M F 3(t), c 3, r 3(t), \Lambda \Lambda$ in turn.

When $c_{n}$ or $r_{n}(t)$ satisfy the given termination condition(the remainder is small enough or becomes a monotonic function), the process terminates, and the decomposition formula is obtained:

$$
s(t)=\sum_{i=1}^{n} I M F_{i}(t)+r_{n}(t)
$$

where $r_{n}(t)$ is called the remainder.

\subsection{Permutation Entropy (PE)}

The permutation entropy of time series $\{x(i), i=1,2, \Lambda, N\}$ can be defined in the form of Shannon entropy as follows: 


$$
H p(m)=-\sum_{g=1}^{m} P_{g} \ln P_{g}
$$

$m$ is the embedding dimension, and $P g$ is the occurrence frequency of each symbol sequence.

When $P_{g}=\frac{1}{m}, H_{p}(m)$ reaches the maximum value $\ln (m !)$, so the permutation entropy $H_{p}(m)$ can be normalized by $\ln (m !)$, that is:

$$
H p=\frac{H p(m)}{\ln (\mathrm{m} !)}
$$

Obviously, the value range of $H p$ is $0 \leq H p \leq 1$. The size of $H p$ represents the degree of randomness of the time series. The larger $H p$ is, the more random the time series is, on the contrary, the more regular the time series is (Yu et al. 2007; Zheng et al. 2013) .

\subsection{MEEMD Method}

Based on the randomness detection of permutation entropy (PE), adopts the MEEMD algorithm. For the non-stationary signal $S(t)$, the decomposition steps of the MEEMD method are as follows (Ramana et al. 2007; Sang 2012; Sang 2018) :

(1) Add white noise signals $n_{i}(t)$ and $-n_{i}(t)$ with zero mean to the original signal $S(t)$, namely:

$$
\begin{aligned}
& S_{i}^{+}(t)=S(t)+a_{i} n_{i}(t) \\
& S_{i}^{-}(t)=S(t)-a_{i} n_{i}(t)
\end{aligned}
$$

Among them, $n_{i}(t)$ represents the added white noise signal, $a_{i}$ represents the amplitude of the added noise signal, here, $i=1,2, \Lambda, N e$, and $\mathrm{Ne}$ represents the logarithm of the added white noise. Perform EMD decomposition on $S_{i}^{+}(t)$ and $S_{i}^{-}(t)$ respectively to obtain the first-order IMF component sequences $\left\{I_{i 1}^{+}(t)\right\}$ and 
86

87

$\left\{I_{i 1}^{-}(t)\right\}(i=1,2, \Lambda, N e)$. The components $I_{1}(t)=\frac{1}{2 N} \sum_{i=1}^{N e}\left[I_{i 1}^{+}(t)+I_{i 1}^{-}(t)\right]$ obtained above are ensemble averaged. Check if $I_{1}(t)$ is abnormal. If the entropy value of the signal is greater than $\theta_{0}$, it is considered to be an abnormal signal, otherwise it is considered to be an approximately stationary signal. After many experiments, it is found that $\theta_{0}$ is suitable for $0.55 \sim 0.6$, this paper takes 0.6 .

(2) If $I_{1}(t)$ is abnormal, continue to perform (1) until the IMF component $I_{p}(t)$ is not an abnormal signal.

(3) Separate the decomposed previous p-1 components from the original signal, namely:

$$
r(t)=S(t)-\sum_{j=1}^{p-1} I_{j}(t)
$$

(4) Then perform EMD decomposition on the remaining signal $r(t)$, and arrange all the obtained IMF components from high frequency to low frequency.

\section{Prediction Method and MDPRM Design}

\subsection{Prediction Method}

The precipitation is a weakly correlated and highly complex nonlinear dynamic system because of affected by comprehensive action of many physical elements such as atmospheric circulation, hydrometeorological elements, and physical geography etc. Its interannual variation does not move in a fixed cycle. Instead, it contains changes and local fluctuations on various time scales, which makes the medium and long-term precipitation prediction is difficult and the accuracy is low (Wang et al. 2022). The core idea of this paper is to use MEEMD to decompose the precipitation data into different decomposition items, and use PSO-SVM, CNN and RNN models for different decomposition items to establish a combined prediction model to reduce the 

prediction error caused by the non-smoothness of the data (Xing et al. 2015).

\subsubsection{PSO-SVM}

(1) Train SVM

a) The initialization constant $\varepsilon=0.01, r=0.05$, the parameters $C$ and $c$ are respectively a random number, the Lagrange multiplier $a=0, a x=0$, and the kernel function matrix $H=0$;

b) Calculate the kernel function $K\left(x_{i}, x_{j}\right)+1, i, j=1,2, \Lambda, l$; the kernel function adopts the RBF kernel function, where $\sigma=2$; namely:

$$
K(x, x i)=\exp \left(-\frac{(x-x i)^{2}}{2 \sigma^{2}}\right)
$$

c) Calculate the error $E i$, where $y i$ is the model predicted value of the $i$

particle:

$$
E i=y i-\sum_{j=1}^{l}\left(a j-a j^{*}\right)(K(x i, x j)+1)
$$

d) Calculate gradients $\delta t a(i)$ and $\delta t a^{*}(i)$ according to formulas (7) and (8), and update $\Delta a i$ and $\Delta a_{i}{ }^{*}$ according to conditions (9) and (10):

$$
\begin{gathered}
\delta a_{i}=-\eta \frac{\overline{\partial L}}{\partial a_{i}}=-\eta\left(-E_{i}+\varepsilon+\frac{a_{i}}{C}\right) \\
\delta a_{i}{ }^{*}=-\eta \frac{\overline{\partial L}}{\partial a_{i}{ }^{*}}=-\eta\left(-E_{i}+\varepsilon+\frac{a_{i}{ }^{*}}{C}\right)
\end{gathered}
$$

Since the optimization variable has a limited range of variation, that is $0 \leq a_{i}, a_{i}{ }^{*}<C \cdot c$, take as follows :

$$
\Delta a i= \begin{cases}-a_{i} & \delta a_{i}<-a_{i} \\ \delta a_{i} & -a_{i} \leq \delta a_{i} \leq C \cdot c-a_{i} \\ C \cdot c-a_{i} & \delta a_{i}>C \cdot c-a_{i}\end{cases}
$$




$$
\Delta a_{i}{ }^{*}= \begin{cases}-a_{i}{ }^{*} & \delta a_{i}{ }^{*}<-a_{i}{ }^{*} \\ \delta a_{i} & -a_{i}{ }^{*} \leq \delta a_{i}{ }^{*} \leq C \cdot c-a_{i}{ }^{*} \\ C \cdot c-a_{i}{ }^{*} & \delta a_{i}{ }^{*}>C \cdot c-a_{i}{ }^{*}\end{cases}
$$

e) Update the $a_{i}$ and $a_{i}{ }^{*}$ according to formulas (11) and (12);

$$
a_{i}=a_{i}+\Delta a_{i}
$$

$$
a_{i}{ }^{*}=a_{i}{ }^{*}+\Delta a_{i}{ }^{*}
$$

f) Determine whether the stop condition is met, if so, terminate the iteration,

147 function. Search the optimal value as the global optimal fitness value, and take its otherwise return c);

g) Output the result yout;

h) Calculate the mean square error according to the following formula, which is the fitness function of the particles in the PSO algorithm:

$$
f i t(i)=\left(\sum_{i=1}^{l}\left(y_{i}-\overline{y_{i}}\right)^{2}\right)^{\frac{1}{2}}, \quad i=1,2, \Lambda, l
$$

Among them, fit $(i)$ represents the fitness value of the $i$ particle; $l$ represents the number of samples; $y_{i}$ represents the model prediction value of the $i$ particle; $\overline{y_{i}}$ represents the model expectation value of the $i$ sample.

\section{(2) POS Optimization Process}

a) Initialize the constant $m, c 1, c 2$, Maxiter, $v \max ;$ b) Take the optimization target parameters $C$ and $c$ as particles, their initial values are the values of the parameters $C$ and $c$ in 1.a) respectively, and take them as the optimal solutions of the current individual; c) Set the mean square error xerr between the particle fitness and the prediction result in the 1.g) as the objective corresponding particle as the current global optimal solution; d) Start the iterative 
optimization process; e) Update the velocity and position vector; f)Re-substitute the updated value of the parameter $C$ and $c$ into the PSO-SVR model, and the SVR training is re-trained according to the process described in (1), save the output results, and calculate the fitness value of the particles ( $\mathrm{Li}$ et al. 2015; Su et al. 2012; Wang et al. 2019).

Establish the SVM prediction model for the precipitation sub-item data respectively, and use the PSO algorithm to find the optimal parameters. Then use the optimal parameters to optimize the prediction model. Finally, use the optimized model to predict the precipitation sub-item data (Zhang et al. 2009; Zhao et al. 2019).

\subsubsection{CNN}

(1) The input data of the convolution layer is the output data of the input layer or the sampling layer. Convolution calculation is performed for each convolution kernel and the features map of the upper layer to obtain the feature map of this layer. The calculation formula of the convolution layer is:

$$
x_{j}^{l}=f\left[\sum_{i \in P_{j}} x_{i}^{l-1} * w_{i j}^{l}+b_{j}^{l}\right]
$$

Among them, $f(\sim)$ is the nonlinear mapping Relu activation function, and its calculation formula is as follows:

$$
f(u)= \begin{cases}u, & \text { if } u>0 \\ 0, & \text { otherwise }\end{cases}
$$

The $P_{j}$ represents the selected window on the input feature, that is, the position on the input feature corresponding to the current convolution kernel in the calculation during the convolution process; $x_{i}^{l-1}$ and $x_{j}^{l}$ are respectively the corresponding value of the $l-1$ input feature and the $l$ output feature. $w_{i j}^{l}$ is the weight value of the 
171 convolution kernel; $b_{j}^{l}$ is the bias of the feature, each layer corresponds to one (Chen 172 et al. 2019).

173 (2) The fully-connected layer can be regarded as a simple single-layer perceptron, 174 which performs fully-connected calculations on all the data in the previous layer. The 175 calculation formula is:

$$
x^{l}=f\left(u^{l}\right), \quad u^{l}=w^{l} * x^{l-1}+b^{l}
$$

Here, $f(\sim)$ is Relu activation function of the nonlinear mapping, $x^{l}$ is the output value of the fully connected layer, $w^{l}$ and $b^{l}$ are the weight values and biases when calculating the $l-1$ layer to the $l$ layer.

(3) Output layer (regression layer): Assuming that the true label corresponding to the $i$ input feature $x_{i}$ in the regression problem is $y^{i}=\left(y_{1}, y_{2}, \Lambda, y_{m}\right)^{\mathrm{T}}$, and $M$ is the total dimension of the label vector, then $l_{t}^{i}$ represents the prediction error ( aka residual) between the network regression prediction value $\left(\hat{y}_{t}^{i}\right)$ and the real value $\left(y_{t}^{i}\right.$ ) in the $t$ dimension on sample $i$ (Xu et al. 2019):

$$
l_{t}^{i}=y_{t}^{i}-\hat{y}_{t}^{i}
$$

The $l_{2}$ loss function for $\mathrm{N}$ samples is defined as follows:

$$
\text { Ll2loss }=\frac{1}{N} \sum_{i=1}^{N} \sum_{t=1}^{M}\left(l_{t}^{i}\right)^{2}
$$

\subsubsection{RNN}

For the time series prediction model, the input and output data are related, which is suitable for the RNN model with memory. Suppose $x, S, o$ be 3 vectors to represent the values of input layer, hidden layer and output layer respectively, $U, V, W$ respectively represent the weight matrices of the input layer and the hidden layer, the hidden layer and the output layer, and the hidden layer and the 
194 hidden layer, then:

$$
s_{t}=f\left(U x_{t}+W s_{t-1}+b_{s}\right)
$$

$$
O_{t}=g\left(V s_{t}+b o\right)
$$

197 In the formula, $s_{t}$ is the hidden state at time $t ; x_{t}$ is the input at time $t ; s_{t-1}$ 198 is the hidden state at time $t, O_{t}$ is the output at time $t ; f$ and $g$ are the 199 excitation functions of the output layer and the hidden layer, $b_{s}$ and $b o$ are the bias 200 of the output layers and the hidden layer (Zaher et al. 2018).

\subsection{MDPRM Design}

Due to the complexity of precipitation forecasting, especially the high frequency components are random variables and non-stationary signals, it is difficult to make accurate forecasts using conventional forecasting methods. This paper combines the

205 forecasting methods in section 3.1, and proposes a new precipitation combined 206 forecasting mode, that is modified ensemble empirical mode decomposition-prediction -reconstruction model (MDPRM) (Altunkaynak et al. 2015). 


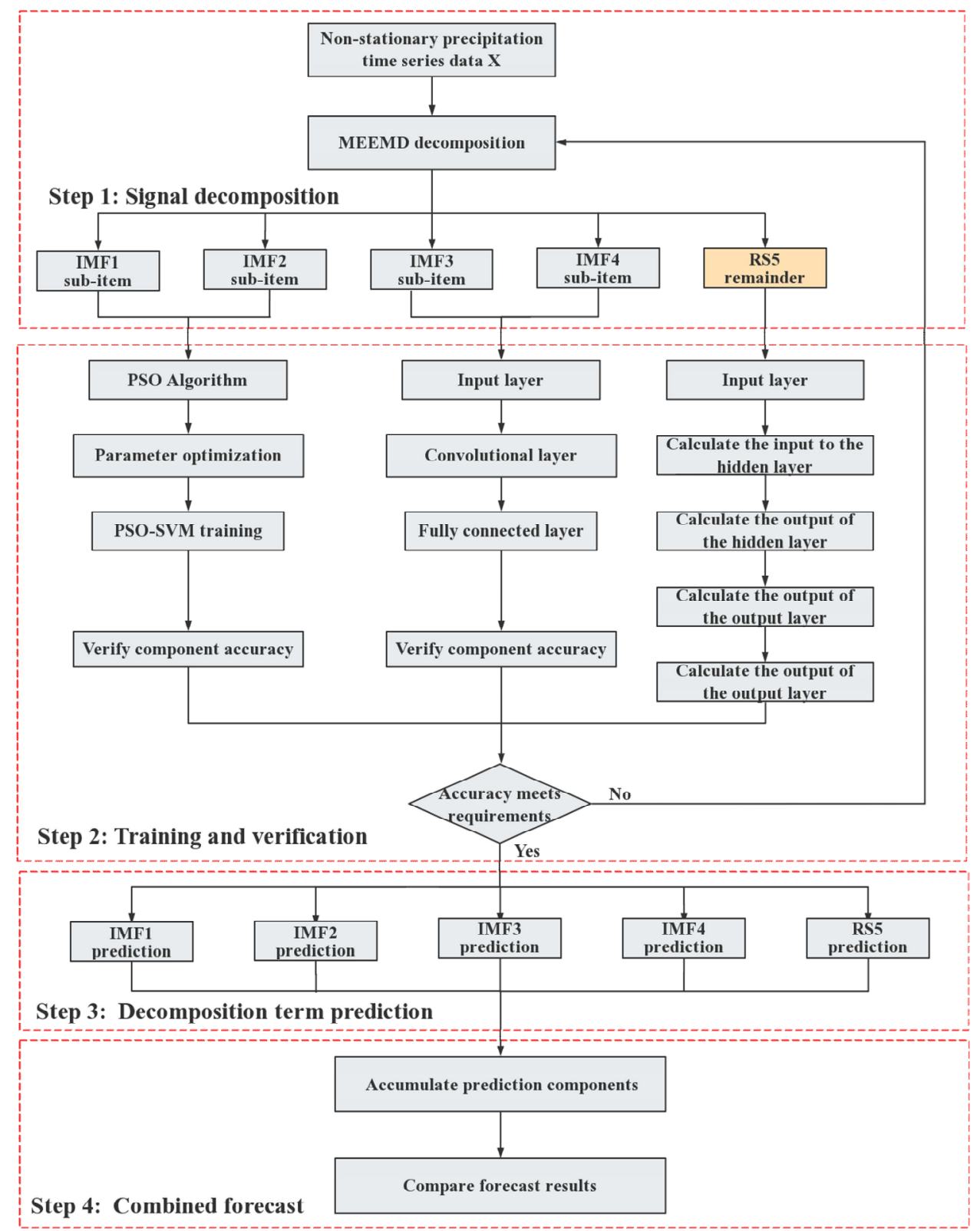

Fig.1 MDPRM model structure

The MDPRM model structure is shown in figure 1. The specific steps of

211 modeling are as follows: (1) Data preprocessing. Complete data storage management,

212 basic analysis of the relationship between the mean and variance of the data, and

213 calculation of the correlation coefficient between the data. Eliminate obviously

214 erroneous data through analysis and obtain a usable sample set; (2) Modified

215 Ensemble Empirical Mode Decomposition (MEEMD) of the original precipitation 
time series; (3) According to each sub-item signals features, the Particle Swarm Optimization Support Vector Machine (PSO-SVM), Convolutional Neural Network $(\mathrm{CNN})$ prediction and Recurrent Neural Network (RNN) prediction are used respectively; (4) The combination of the predicted values of each component and the evaluation and feedback of the predicted results.

\subsection{Prediction Results Evaluation}

\subsubsection{Prediction Accuracy Evaluation}

Some of the measured data are used as training samples to calibrate the parameters of each algorithm, and the rest of the measured data are used as test samples and prediction results to compare the prediction effects of each algorithm. The relative error(e), mean absolute percentage error $(M A P E)$, root mean square error $(R M S E)$ and consistency index $(\alpha)$ are used to evaluate the simulation accuracy. The smaller the mean absolute percentage error(MAPE) and the root mean square error $(R M S E)$, the higher the simulation accuracy; the larger the consistency index $(\alpha)$, the closer to 1 , the higher the simulation accuracy ${ }^{[34]}$.

\subsubsection{Z-Index Drought Evaluation}

The Z-index is one of the most widely used indices. To calculate the Z-index, it is assumed that the precipitation in a certain period follows the Person-III distribution. After normalizing the precipitation, the probability density function can be converted operation. The Z-index method assumes that the rainfall in a certain period of time obeys the Person-III distribution, and as the time series grows, the precipitation usually obeys or close to the normal distribution. When calculating the Z-index value, the deviation coefficient is used. When the standard variable $\varphi i$ is determined, the Z-index value mainly depends on the deviation coefficient $C s$. The Z-index value is not only related to the precipitation, but also to the distribution characteristics of 
precipitation in the region. (1) $\mathrm{Z}>1.645$, severe waterlogging; (2) $1.037<\mathrm{Z} \leq 1.645$,

242

243

244 heavy waterlogging; (3) $0.842<\mathrm{Z} \leq 1.037$, partial waterlogging; (4) $-0.842 \leq \mathrm{Z} \leq 0.842$, normal; (5)- $1.037 \leq \mathrm{Z} \leq-0.842$, partial drought; (6) -1.645 $\leq \mathrm{Z} \leq-1.037$, heavy drought; (7) $\mathrm{Z}<-1.645$, severe drought (Zhang et al. 2018).

\section{Verification of Predictive Model}

\subsection{Data Overview}

The data in this paper come from the Guizhou Meteorological Bureau, which is accurate and credible. As shown in Figure 2, the annual average precipitation in the summer from 1961 to 2018 in the Wujiang River Basin was $518.66 \mathrm{~mm}$. The minimum value appeared in 1972 , which was $256.92 \mathrm{~mm}$; the maximum value appeared in 1998 , which was $676.36 \mathrm{~mm}$. According to the trend value curve, it can be seen that the annual average precipitation in the Wujiang River Basin presents an increasing trend, with an average annual increase of $0.5691 \mathrm{~mm}$. According to the 5-year moving average curve, it can be seen that in the 51 years from 1961 to 2018, the average summer precipitation in the Wujiang River Basin has roughly experienced a cycle of abundance $\rightarrow$ dry $\rightarrow$ abundance $\rightarrow$ dry.

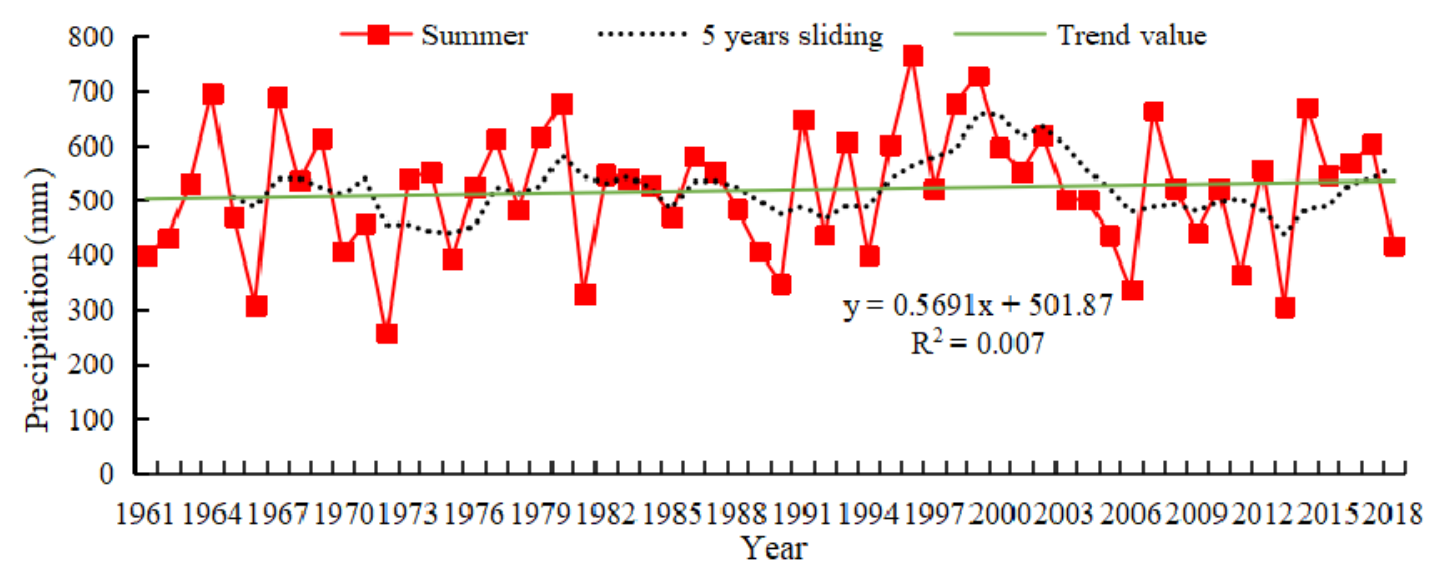

Fig.2 Seasonal precipitation in the Wujiang River Basin (1986-2018)

\subsection{Implementation of Predictive Model}


4.2.1 MEEMD Decomposition of Precipitation Time Series

The seasonal precipitation series in the Wujiang River Basin has obvious nonlinearity and non-stationarity. In order to better local analysis of precipitation time series to obtain higher prediction accuracy, this paper uses MEEMD to decompose the precipitation sample data for 58 summers' from 1961 to 2018 in the Wujiang River Basin. During decomposition, the intensity of white noise is set to 0.2 , the maximum number of natural modes is 6 , the number of adding noise is 30 , the embedding dimension is 6 , and the time delay is 1 . When the permutation entropy $\theta 0$ of the signal takes 0.55 , the precipitation original signal $\mathrm{x}$ is decomposed into IMF1 IMF3 and the remainder RS4; when the permutation entropy $\theta 0$ of the signal takes 0.6 , it is decomposed into IMF1 IMF4 and the remainder RS5, Figure 3 shows the MEEMD decomposition result of precipitation (Erhao et al. 2015). 
Decompose 4 layers, $\theta 0=0.55$
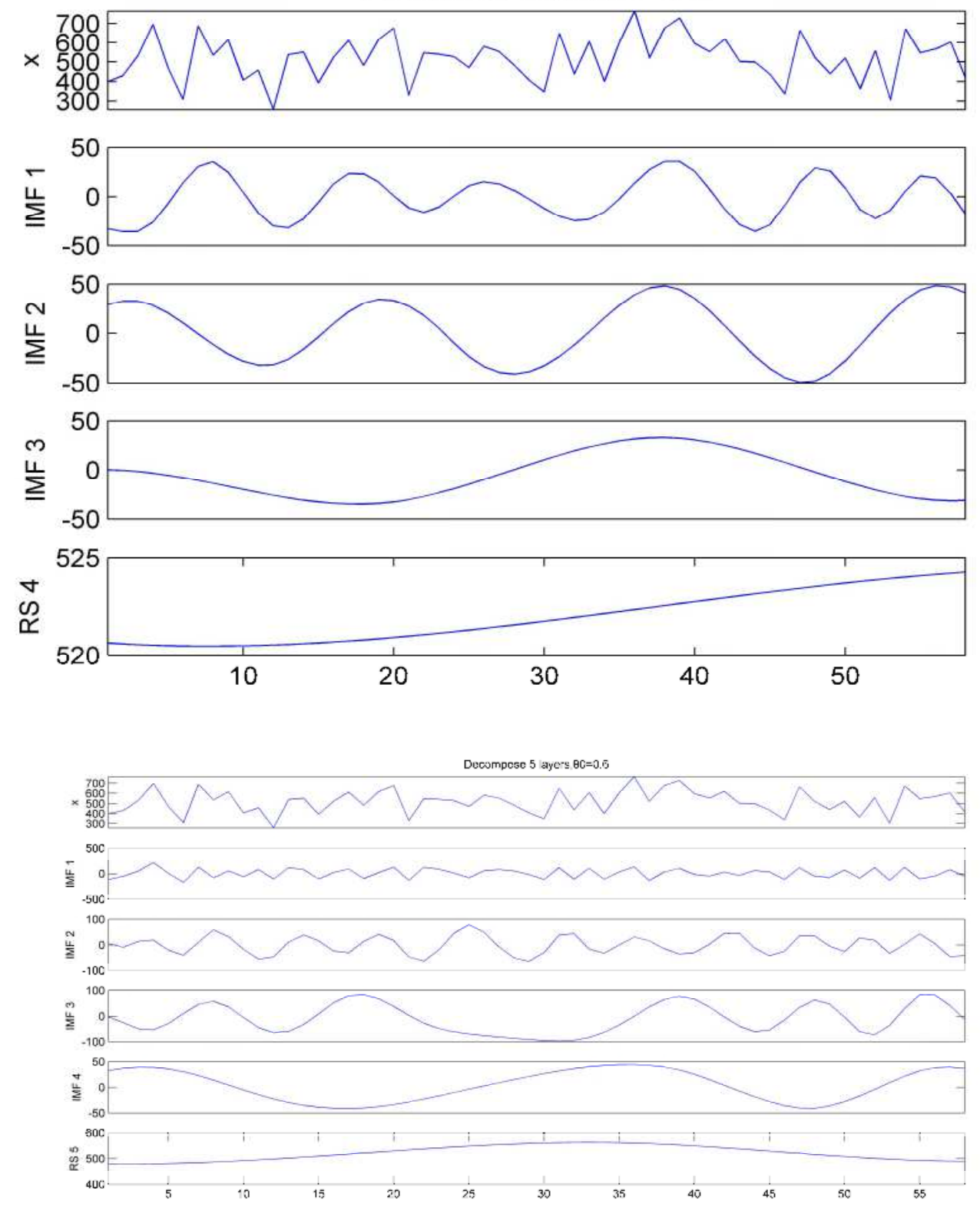

The difference between the original signal and the reconstructed signal

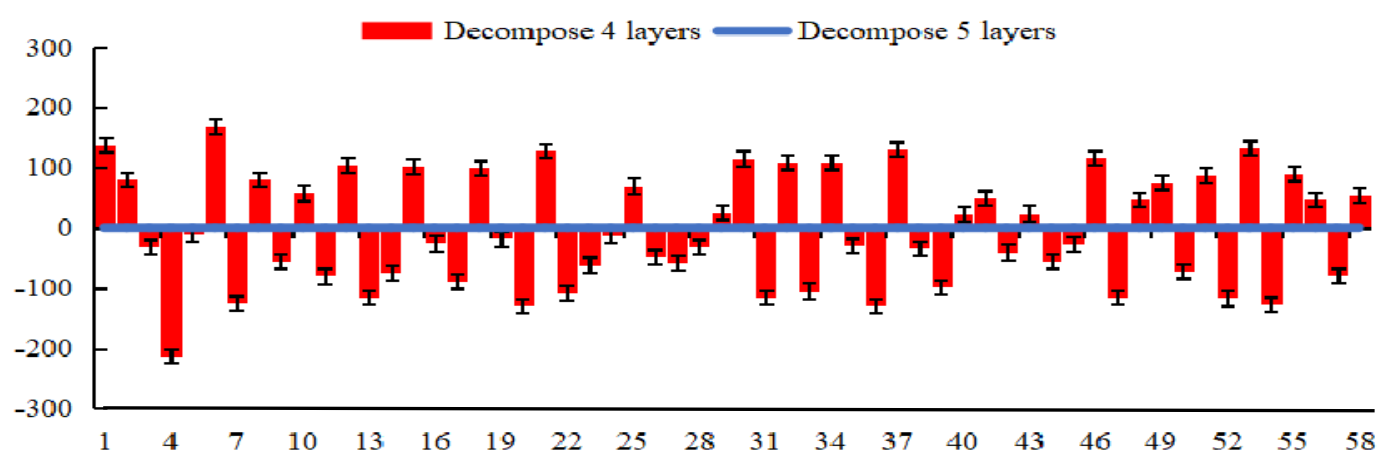

273 Fig. 3 The MEEMD decomposition result of precipitation 
The calculation shows that the reconstruction error of MEEMD 4-layer decomposition is -2.82 , while the magnitude of reconstruction error amplitude of MEEMD 5-layer decomposition is less than $4^{-13}$, which indicates that MEEMD 5-layer decomposition is very consistent with the actual composition of the original signal, so this paper adopts 5-layer decomposition. The decomposition items are screened according to the root mean square error index. The decomposition items of IMF1 and IMF2 change rapidly with time, and the particle swarm optimization support vector machine (PSO-SVM) will be used to predict. Due to the large fitting error of the decomposition terms of IMF3 and IMF4, CNN is used to predict them, while the RNN model is used to predict the remaining term RS5.

\subsubsection{PSO-SVM Prediction（IMF1、IMF2）}

Parameter initialization: learning factors $(\mathrm{c} 1=1.5 ; \mathrm{c} 2=1.7) ;$ maximum evolutionary number (maxgen=200); maximum population size (sizepop=20); initial value of $k(k=0.6)$, the relationship between rate and $x(V=k X)$; the elastic coefficient in front of the velocity in the rate update formula $(\mathrm{wV}=1)$; the elastic coefficient in front of the velocity in the population update formula ( $\mathrm{wP}=1$ ); the Cross Validation parameter $(\mathrm{v}=5)$; the maximum value of the change of parameter $\mathrm{c}($ popcmax $=100)$; the minimum value of the change of parameter $\mathrm{c}($ popcmin=1); the maximum value of the change of parameter $g$ (popgmax0.5); the minimum value of the change of parameter $g$ (popgmin=0.3); the population size $(\mathrm{pop}=500)$. Figure 4 shows the comparison between the predicted and actual values of IMF1 IMF2. 

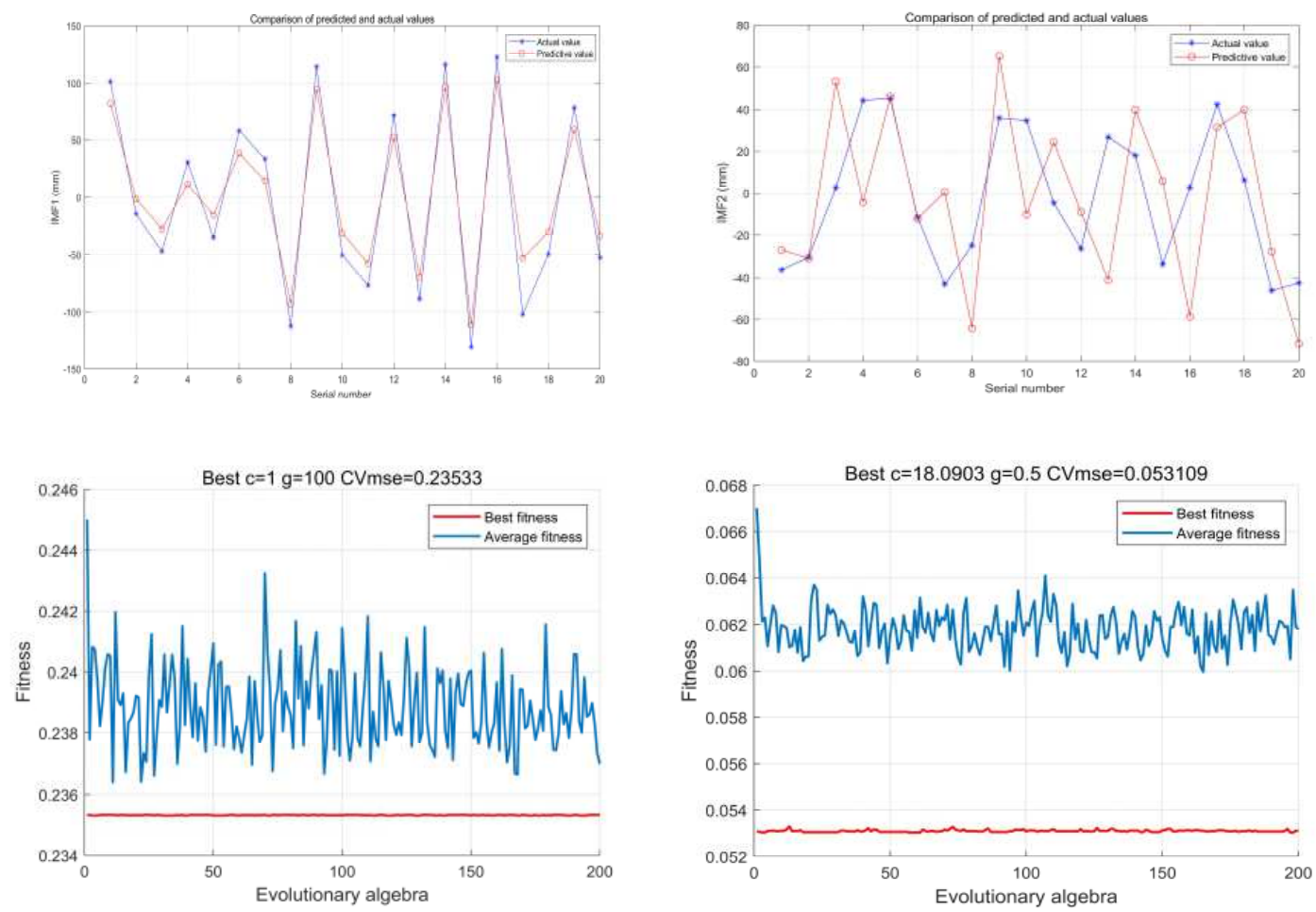

Fig.4 Comparison between the predicted and actual values of IMF1 IMF2

\subsubsection{CNN Prediction（IMF3、IMF4）}

The convolutional neural network is constructed with 3 layers: 1 input layer, 1 convolutional layer and 1 fully connected layer. The input samples are 55 groups of $2 \times 2 \times 13 \mathrm{D}$ data, and the convolutional layer is set to 4 convolution kernels respectively. The size of the convolution kernel is $1 \times 1$. The output of the convolution layer is used as the input of the fully connected layer to form a single-layer perceptron and output the predicted value.

(1) Input layer: The size of the input layer is $2 \times 2 \times 1$; (2) Convolution layer: Since the matrix of the input data is small, a smaller convolution kernel $1 \times 1$ is selected. The number of convolution kernels is 4 , and the step size is 1 . In order to prevent the matrix losing too much information during the convolution process, Padding ='same' is used for zero-padding. At the same time, set the step size of convolution to 1; (3) Batch normalization layer: use mean variance method to normalize batches; (4) 

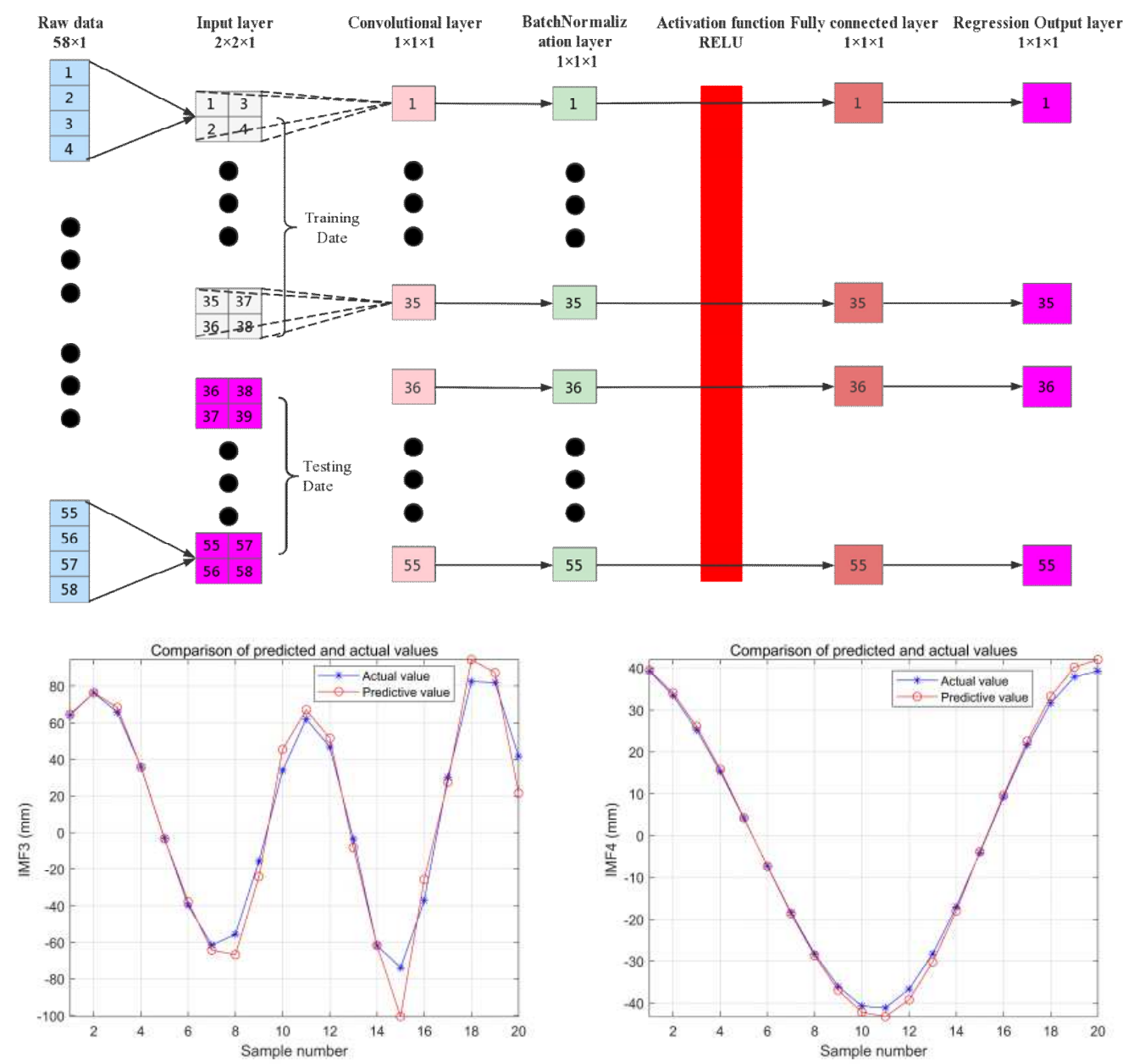

Fig. 5 Comparison between the CNN network model and IMF3 IMF4

\subsubsection{RNN Neural Network Prediction (RS5)}

The RNN model is used to predict the decomposition item RS5. The original 
time series data with a structure of $58 \times 1$, and the data sequence is processed into $55 \times 10$ by statement $[$ in, out $]=$ create_serino $($ lie_samp, 10$)$.Select the first 10 columns of sample data for correlation analysis, it shows that the correlation coefficient of the

321 first 8 columns of sample data is greater than 0.5 , which is a strong correlation, in 322 other words, 50 years can represent the approximate distribution of the entire series. Given a precipitation time series input data $x=\left(x_{1}, x_{2}, \Lambda x_{t}, \Lambda x_{49}\right)$, output data $y=x_{50}$, and the sequence roughly goes through a cycle of 5 years. The input data is further processed into $9 \times 5 \times 49$ through data preprocessing, normalization and structuring, and the output layer is $9 \times 1$. Establish the network structure as dimensions, the number of neurons in the input layer is 9 , the number of neurons in the hidden

328 layer is 10 , the number of neurons in the output layer is 1 , the activation function is 329 sigmoid, learning rate $=0.001$, Training Step=5000, batch $=20$, activation 330 functions=sigmoid; the learning rate algorithm is RAdam; Figure 6 shows the 331 structure of the recurrent neural network and the comparison between the RS5 332 predicted value and the actual value.
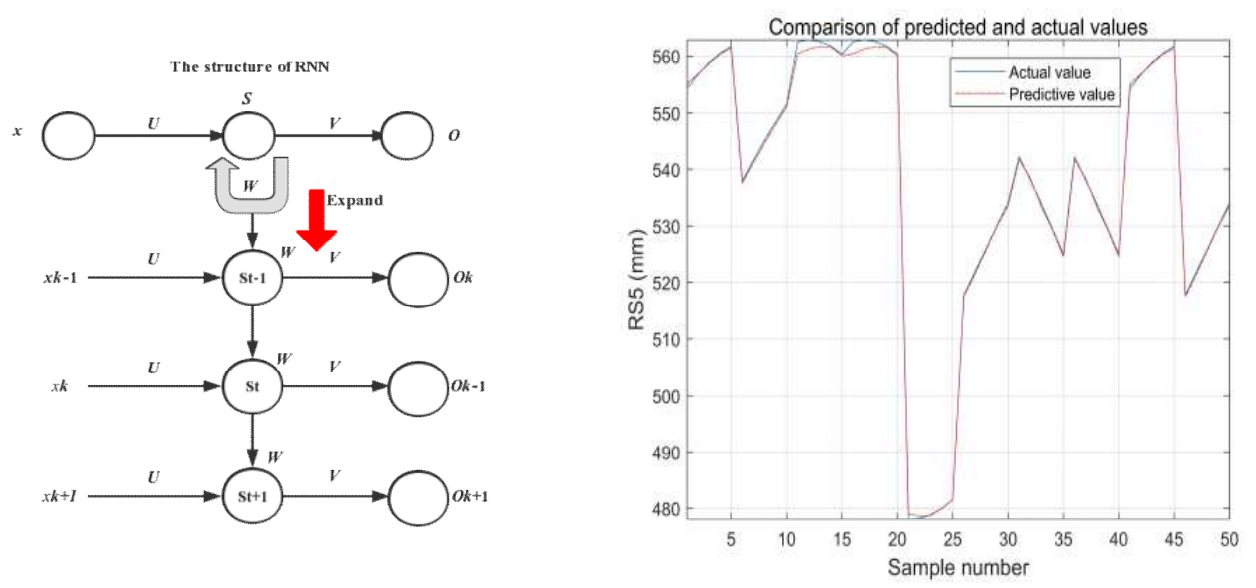

Fig.6 The structure of RNN and the comparison between the RS5 
settings, the first 38 years of summer precipitation data in the Wujiang River Basin from 1961 to 2018 were used for training, and the latter 20 sets of data were simulated, and the simulation results were compared with the measured data and analyzed ${ }^{[30]}$. Figure 7 shows the comparison of the simulation results of summer precipitation changes in the Wujiang River Basin.

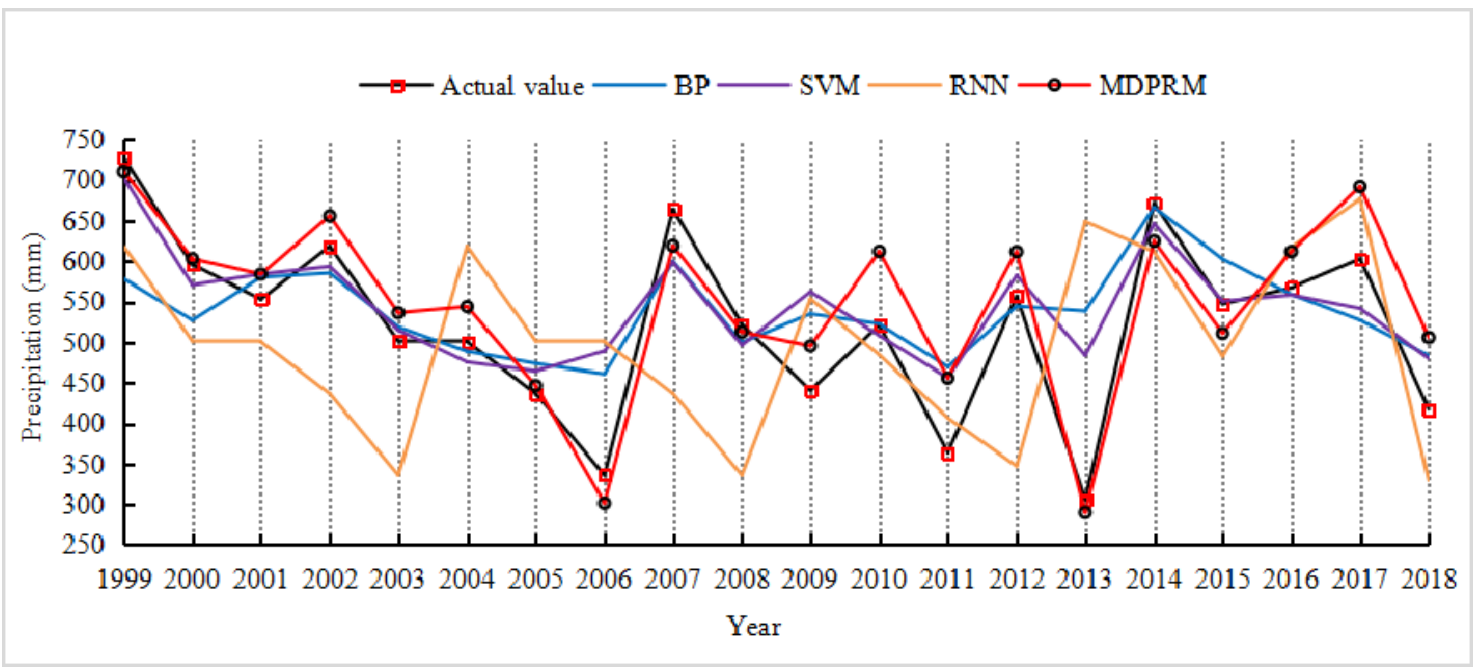

Fig.7 Comparison of the simulation results of summer precipitation changes

Overall, the qualification rate of the four forecast models is $100 \%$ (the absolute percentage error is less than $20 \%$ as the qualification standard $)^{[20]}$. It can be seen intuitively from figure 7 that the prediction result of the MEEMD combined model in this paper is closer to the real value and has better prediction accuracy. For a more comprehensive evaluation of model performance, table 1 presents the model performance statistical values MAPE、RMSE $\alpha$ and $\Omega$. It can be seen from table 1 that the MAPE of MEEMD decreased from 0.14 to 0.09 , and the RMSE decreased from 0.37 to 0.30 ; and the $\alpha$ increased from 0.53 to 0.75 significantly. It can be concluded that the three performance evaluation indicators of the MDPRM model are significantly better than the other three models, and it has more accurate time series data prediction ability.

Table 1 Simulation Accuracy Evaluation of Different Algorithms 


\begin{tabular}{ccccc}
\hline Serial Number & Algorithms & MAPE & RMSE & $\alpha$ \\
\hline 1 & BP & 0.14 & 0.37 & 0.53 \\
2 & SVM & 0.12 & 0.35 & 0.64 \\
3 & RNN & 0.26 & 0.51 & 0.33 \\
4 & MDPRM & 0.09 & 0.30 & 0.75 \\
\hline
\end{tabular}

\subsection{Application of Precipitation Prediction Results}

The MEEMD decomposition of the summer precipitation in the Wujiang River

Basin from 1961 to 2018 was carried out, and the combined prediction model was used to predict the precipitation IMF1, IMF2', IMF3', IMF4 and $\mathrm{RS}^{\prime}$ in the next 10

359 years, and the precipitation forecast value $S$ can be obtained by

$360 \mathrm{~S}=\mathrm{IMF1}+\mathrm{IMF} 2^{\prime}+\mathrm{IMF} 3^{\prime}+\mathrm{IMF} 4^{\prime}+\mathrm{RS5}^{\prime}$ accumulation. The predicted results are

361 shown in table 2. According to the prediction results, the Z-index was used to analyze

362 the characteristics of drought grades in the Wujiang River Basin in the next 10 years.

363 Table 2 Summer precipitation forecast and drought assessment of Wujiang River

364 Basin in the next 10 years

\begin{tabular}{|c|c|c|c|c|c|c|}
\hline \multirow{2}{*}{$\begin{array}{c}\text { Decomposition } \\
\text { Label }\end{array}$} & \multirow{2}{*}{$\begin{array}{c}\text { Prediction } \\
\text { Method }\end{array}$} & \multicolumn{5}{|c|}{ Years } \\
\hline & & 2019 & 2020 & 2021 & 2022 & 2023 \\
\hline IMFI' & PSO-SVM & -0.09 & -0.09 & -0.09 & -0.09 & -0.09 \\
\hline IMF2' & PSO-SVM & 5.09 & -7.04 & -6.85 & 0.11 & -6.15 \\
\hline IMF3' & $\mathrm{CNN}$ & -53.30 & -64.53 & -50.68 & -24.92 & -1.26 \\
\hline IMF4 & $\mathrm{CNN}$ & 33.07 & 27.04 & 19.43 & 9.54 & 0.47 \\
\hline RS5' & $\mathrm{ABC}-\mathrm{BP}$ & 488.51 & 485.56 & 485.77 & 489.00 & 493.07 \\
\hline \multicolumn{2}{|c|}{ Predicted results $(\mathrm{mm})$} & 473.29 & 440.94 & 447.58 & 473.64 & 486.05 \\
\hline \multicolumn{2}{|c|}{$\mathrm{Z}$ index } & -0.35 & -0.65 & -0.59 & -0.34 & -0.23 \\
\hline
\end{tabular}




\begin{tabular}{|c|c|c|c|c|c|c|}
\hline \multicolumn{2}{|c|}{ Drought grade } & Normal & Normal & Normal & Normal & Normal \\
\hline \multirow{2}{*}{$\begin{array}{c}\text { Decomposition } \\
\text { Label }\end{array}$} & \multirow{2}{*}{$\begin{array}{l}\text { Prediction } \\
\text { Method }\end{array}$} & \multicolumn{5}{|c|}{ Years } \\
\hline & & 2024 & 2025 & 2026 & 2027 & 2028 \\
\hline IMFI' & PSO-SVM & -0.09 & -0.09 & -0.09 & -0.09 & -0.09 \\
\hline IMF2' & PSO-SVM & 2.03 & 2.44 & 1.85 & -5.38 & -2.11 \\
\hline IMF3' & $\mathrm{CNN}$ & 10.70 & 9.25 & -0.54 & -9.73 & -10.83 \\
\hline IMF4' & $\mathrm{CNN}$ & -8.58 & -17.32 & -24.53 & -30.55 & -35.04 \\
\hline RS5 & $\mathrm{ABC}-\mathrm{BP}$ & 493.87 & 493.01 & 493.18 & 489.20 & 492.24 \\
\hline \multicolumn{2}{|c|}{ Predicted results(mm) } & 497.94 & 487.30 & 469.87 & 443.45 & 444.18 \\
\hline \multicolumn{2}{|c|}{ Z index } & -0.12 & -0.22 & -0.38 & -0.63 & -0.62 \\
\hline \multicolumn{2}{|c|}{ Drought grade } & Normal & Normal & Normal & Normal & Normal \\
\hline
\end{tabular}

\section{Summary}

(1) By setting the intensity of white noise to 0.2 , the maximum number of natural modes to 6 , the number of adding noise to 30 , the embedding dimension to 6 , the time delay to 1 , and the permutation entropy of the signal to 0.6 , the MEEMD method is used to decompose the original signal $x$ of the 58-year(1961-2018) summer precipitation in the Wujiang River Basin into IMF1 IMF4 and the remainder RS5, which can accurately identify the hierarchical characteristics of the precipitation data,

373 and effectively suppress the modal aliasing phenomenon in the EMD decomposition 374 process, and reduce reconstruction errors.

(2) In this paper, a new precipitation MDPRM is proposed, that is, according to 376 the prediction accuracy and prediction efficiency, particle swarm optimization support 377 vector machine (PSO-SVM) is used to predict the decomposition items IMF1 and 378 IMF2; CNN is used to predicts the decomposition items IMF3 and IMF4; RNN is 
used to predicts the remainder RS5, and the precipitation forecast value is obtained by accumulating the forecast values of each sub-item. Compared with BP, SVM, and RNN, the MAPE of MDPRM is reduced from 0.14 to 0.09 , and the RMSE is reduced from 0.37 to 0.30 ; and the $\alpha$ is significantly improved from 0.53 to 0.75 , indicating that the MDPRM constructed in this paper improves the prediction accuracy.

(3) According to the prediction results of MDPRM, the average summer precipitation in the Wujiang River Basin from 2019 to 2028 is $466.42 \mathrm{~mm}$, the precipitation in 2020 is the smallest at $440.94 \mathrm{~mm}$, and in 2024 is the largest at 497.94mm. Further evaluation of agricultural drought level by Z-index shows that the summers in the 10 years are normal years. According to the prediction results of precipitation and the analysis results of drought characteristics, we should do a good job in the scheduling configuration and efficient use of water resources in summer to maximize economic, social and ecological benefits.

\section{Declarations}

Ethical Approval All authors kept to the Ethical Responsibilities of Authors.

\section{Consent to Participate Not applicable.}

Consent to Publish The authors declare that they consent to publish this manuscript. Authors Contributions YTW: Writing and editing manuscript. JL:Conceptualization, Methodology. RL: Methodology, Data curation. XYS: Writing-original draft, Formal analysis. EHL:Investigation, and Model fitting.

Funding This work was supported by the Guizhou Provincial Science and Technology Department Project (Grant numbers [2021] 4 and [2021] General 469).

Competing Interests The authors declare that they have no conflict of interest. 
Availability of data and materials All the research data are taken from the Guizhou

Meteorological Bureau.

\section{References}

Altunkaynak, Abdusselam, and T. A. Nigussie (2015) Prediction of daily rainfall by a hybrid wavelet-season-neuro technique. Journal of Hydrology 529:287-301.

Chen J, Long Li, Duan Y (2015) Climate analysis of nearly 53a extreme precipitation events in guizhou. Guizhou Meteorol 04:12-15.

Cheng J, Wang J, Hui L (2018) An improved EEMD method and its application in rolling bearing fault diagnosis. Journal of Vibration and Shock 37(16):51-56.

Chen S, Xue Y (2019) Short-term Bus Passener Flow Prediction Based on Imroved Convolutional Neural Network. Comput Sci 5(46):176-177.

Erhao Meng, Shengzhi Huang, Qiang Huang, Wei Fang, Hao Wang (2021) A Hybrid VMD-SVM Model for Practical Streamflow Prediction Using an Innovative Input Selection Framework. Water Resources Management 35:1321-1337.

Jiang Y, Yang X, He F, Chen L, He Z (2016) Super-short-Time Wind Power Forecasting Based on EEMD-IGSA-LSSVM. Journal of Hunan University (Natural Sciences) 43(10)70-78.

Li D, Xue H (2018) Medium and long-term precipitation prediction based on hybrid model. Comput Sci 45(9):275-276.

Li T, Zeng X (2015) Water and sand simulation of yanhe river basin based on particle swarm optimization support vector machine. J Bas Sci Eng 23(7):80-86.

Qi Ouyang, Wenxi Lu, Xin Xin, Yu Zhang, Weiguo Cheng, Ting Yu (2016) Monthly Rainfall Forecasting Using EEMD-SVR Based on Phase-Space Reconstruction. 
428

429

430

Ramana, R. V. , Krishna, B. , Kumar, S. R. , \& Pandey, N. G. . (2013). Monthly rainfall prediction using wavelet neural network analysis. Water Resources Management, 27(10).

Su C, Xiang N, Chen G, Wang F (2012) Water quality evaluation model based on artificial swarm algorithm and BP neural network. Chinese journal of environmental engineering $6.02: 700-703$.

Sang Y (2012) A Practical Guide to Discrete Wavelet Decomposition of Hydrologic Time Series. Water Resour Manage 26:3345-3365.

Sang Y, Li X, Xie P, Liu Y (2018) Universal framework for hydrological time series probabilistic forecasting. Journal of Lake Sci 30(3):611-618.

Wang Y, Liu J, Li R, Suo X, Lu E (2022) Medium and Long-term Precipitation Prediction Using Wavelet Decomposition-prediction-reconstruction Model. Water Resour Manag $\quad 37: 1473-1483$.

Wang Y, Mu H, Xu G (2019) Prediction of photovoltaic cell output power based on improved particle swarm optimization support vector machine. Electr Autom $3: 63-65$.

Xu G, Zhu J, Si C, Hu W, Liu F (2019) Combined Hydrological Time Series Forecasting Model Based on CNN and MC. Computers and Modernization $11: 23-27$

Yu D, Cheng J, Yang Y (2007) Hilbert-Huang transform method for mechanical fault diagnosis . Beijing: Science Press 04:11-25 .

Zaher Mundher Yaseen, Minglei Fu, Chen Wang (2018) Application of the Hybrid Artificial Neural Network Coupled with Rolling Mechanism and Grey Model Algorithms for Streamflow Forecasting Over Multiple Time Horizons. Water 
453 Zhang H, Yang J, Wang Y, Zhou H (2014) Research on the evolution characteristics 454 and prediction model of regional drought in Guizhou. Beijing: China Water 455 Conservancy and Hydropower Press 11:42-59.

456 Zhang X, Chen G, Zhou J, Ma A, Zhang Z (2009) Medium and long-term load 457 forecast based on PSO-RSVR. Power System Protection and Control $458 \quad 37(21): 77-81$.

459 Zhao W, Wang J, Shang M, Liu Y (2019) Pollen concentration prediction model based 460 on particle swarm optimization and support vector machine. Comput Appl $461 \quad 39(01): 104-110$.

462 Zheng J, Cheng J, Yang Y (2013) Modified EEMD algorithm and its applications. 463 Journal of Vibration and Shock 32(21):22-26.

464 Xing Z, Guo H, Fu Q (2015) Analysis of Influencing Factors of Rainfall in 465 Irrigation Area and Combining Rainfall Forecasting. Transactions of the 466 Chinese Society for Agricultural Machinery 46(8): 96-103. 